\title{
On system behaviour using complex networks of a compression algorithm
}

\author{
David M. Walker, ${ }^{1}$ Debora C. Correa, ${ }^{1}$ and Michael Small ${ }^{1,2}$ \\ ${ }^{1)}$ School of Mathematics $\&$ Statistics, University of Western Australia, Nedlands, \\ Perth WA 6009 Australia \\ 2) Mineral Resources, CSIRO, Kensington, Perth WA 6151 Australia
}

(Dated: 8 May 2018)

\begin{abstract}
We construct complex networks of scalar time series using a data compression algorithm. The structure and statistics of the resulting networks can be used to help characterize complex systems and one property, in particular, appears to be a useful discriminating statistic in surrogate data hypothesis tests. We demonstrate these ideas on systems with known dynamical behaviour and also show that our approach is capable of identifying behavioural transitions within EEG recordings as well as changes due to a bifurcation parameter of a chaotic system. The technique we propose is dependent on a coarse grained quantization of the original time series and therefore provides potential for a spatial scale-dependent characterization of the data. Finally the method is as computationally efficient as the underlying compression algorithm and provides a compression of the salient features of long time series.
\end{abstract}

PACS numbers: 05.45.Tp; 89.75.-k

Keywords: Time series; Complex networks; Compression algorithms; Surrogate tests

Large-scale time series data as a form of Big Data is ubiquitous and is becoming the norm. The size of such data sets poses challenges to successfully process, analyze and interpret information contained within the data. Complex networks representations of time series data have been shown to be compact summaries of the data capable of resolving complicated and hidden interactions through their structural properties. In this paper we interpret the compact description of largescale time series data produced by a compression algorithm as a complex network. Properties of this so-called compression network exhibit different features depending on the character of the original time series. That is, their properties appear capable of distinguishing different dynamical behaviours. Furthermore, we demonstrate that a particular property of the compression networks, namely the proportion of network nodes with no connections, is a useful discriminating statistic in hypothesis tests for nonlinear determinism.

\section{INTRODUCTION}

Our research is motivated by the difficulty traditional data processing approaches have in identifying and extracting useful information from large time series. The difficulty of providing full and accurate analysis of large time series data sets provide serious challenges in both industry and government. It is crucial to develop new techniques of data representation to improve both processing accuracy and efficiency. As collecting data becomes increasingly cheap and data sources more plentiful, data analysis techniques increasingly need to be able to rapidly extract useful information from large amounts of data.
We develop a network technique which compresses the representation of large scalar time series while preserving essential properties of the system. A complex network representation has the potential to achieve this and as such motivates a simple question: What does a complex network representation of data compression look like? We make the first strides towards addressing this query and show in this paper that a basic network representation of a compression algorithm provides some useful benefits not a priori anticipated. We demonstrate that not only can the structure of the resulting networks help capture behavioural change in nonlinear systems, but we also find a basic quantity arising from the compression algorithm that can be used as a useful discriminating statistic in standard surrogate data hypothesis tests.

Complex systems and time series measurements describe relationships of interconnected entities in a wide range of domains, including climate models ${ }^{1}$, finance ${ }^{2}$, mining $^{3}$, biological systems ${ }^{4}$ and ecology ${ }^{5}$. The theoretical and computational study of complex system time series as complex networks has gained attention and traction in recent years. Methods include pseudo-periodic networks ${ }^{6}$, visibility graphs ${ }^{7,8}$, phase space networks ${ }^{9}$, recurrence networks ${ }^{10,11}$ and ordinal partition networks ${ }^{12}$ to name but a selection of the approaches. In such networks the variety of entities (for various different definitions of "entities") describing a complex system is modelled as nodes in the network, while the intrinsic relationship among interconnected entities are modelled as edges. The patterns of such interactions have proven to be a key aspect when uncovering interesting properties of the studied systems ${ }^{13}$. These approaches have been primarily concerned with questions on how much information of the original time series is preserved in the network representation, and how system properties can be extracted or reconstructed from a network representation. In this paper we develop a novel representation of time series data from the perspective of compression, to 
produce complex networks capable of capturing key and essential features of the original system which are present in the time series.

The network representations produced by this method provide - at the chosen quantization level — a parameter-free characterization of an optimal compression of the coarse-grained signal. Unlike previous network-from-time-series representation techniques, the resulting representation is both adaptive and of variable length. More complex dynamics will be represented by more intricate heterogeneous networks, simpler (usually periodic) dynamics will correspond to bland and homogeneous networks.

Alternatively, compression of large-scale data proves useful for both storage and transmission. Compression can be best achieved if there is some predictability within the data. This feature has been exploited by researchers in nonlinear time series analysis by using principles such as Rissanen's minimum description length ${ }^{14}$ to discriminate among competing models within a given class ${ }^{15,16}$. Dynamical models are reconstructed and the total code length of the model parameters, their precisions and the model prediction errors are compared between models and the raw data itself. Models with minimum code, or description length, are preferred and have been shown to be useful for prediction and system characterization ${ }^{15,16}$. In this paper we take a different tack and instead consider the structure inherent in the output of the compression algorithms.

In particular we consider Lempel-Ziv ${ }^{17}$ stream codes which are universal data compression schemes, i.e., they are designed to provide reasonable compression for any source $^{18}$. Consider a time series which has been symbolically encoded, e.g., data values are replaced by a 0 or a 1 if they are below or above the data median, respectively. Lempel-Ziv compression works by replacing substrings of 0's and 1's by pointers to already seen substrings, i.e., codewords. A dictionary of codewords is progressively built up as novel substrings appear in the data. Compression can be achieved as the original data is replaced by a time series of pointers to codewords and, combined with the dictionary of codewords, the total code length may be shorter than the code length of the original data sequence.

Here, we recognize that the time series of codeword pointers induces a complex network which is a directed graph. The network nodes represent the individual codewords and two nodes are connected if the codewords follow each other in the codeword sequence. We can also keep track of the number of times one codeword follows another to find a weighted directed network, or simply ignore this count and also the directionality to study the structure of the resulting undirected network. Here we choose to do the latter, and we show that basic network statistics and measures of the resulting compression network can be used to detect behavioural change for time series of deterministic systems.

We note that within the dictionary of discovered code- words some are never seen again in the time series and so are not "emitted" in the sequence of codewords. In this case, such prototype codewords are isolated nodes in the induced compression network. We suggest and demonstrate here that a simple count of these unused codewords appears to be a useful discriminating statistic in surrogate data testing despite it not being a pivotal statistic $^{19,20}$.

The general idea of surrogate data, originally developed by Theiler and colleagues ${ }^{19,21}$, is to formalize a framework in which one can look for explanations about the variability of a time series. As variability can be due to many factors (chaos, linear correlation, noise, nonlinearity, etc), the aim of surrogate methods is to justify the need of a nonlinear analysis when one can discard, with some level of confidence, simple explanations about the data. Three null hypotheses proposed by Theiler and commonly referred to as Algorithm 0, 1 and 2 consider in increasing level of complexity that the observed data is consistent with (i) i.i.d noise, (ii) linearly filtered i.i.d noise, and (iii) a monotonic static nonlinear transformation of linearly filtered noise, respectively.

Surrogates, in the form of realizations, constrained or unconstrained, consistent with each null hypothesis and the observed data are generated and a test statistic is calculated. If the statistic calculated for the observed data is, at some significance level, statistically different from the distribution of values of the statistic calculated for the surrogate data, then there is evidence to reject the null hypothesis. One then considers a more complicated null hypothesis or starts to build nonlinear models. If the surrogate data realizations are unconstrained (e.g., generated from a AR model of the data for Algorithm 1 tests) then a pivotal test statistic is required. If the surrogate data realizations are constrained (e.g., preserving a property of the data such as autocorrelation structure in Algorithm 1 tests) then one can use a statistic that is not pivotal. Since our proposed statistic - the number of unused codewords - is not a dynamical invariant, it is unlikely to be pivotal ${ }^{22}$ and so in the following we consider constrained realizations for Algorithm 0, 1 and 2 surrogate data tests.

The remainder of the paper is organized as follows: Section II describes our protocol for converting a time series to a complex network by using a compression algorithm. In Section III we establish the usefulness of our proposed discriminating statistic for identifying possible nonlinear behaviour compared to a linear null hypothesis and investigate its robustness to noise and symbolization. We further apply the method to EEG data where behavioural transitions have been identified to demonstrate that the proposed statistic is practicable. We then describe how properties of the structure of the compression networks can capture different types of system behaviour and can be used to distinguish random verses chaotic dynamics. We close the paper with a summary of the merits of our proposed approach. 


\section{COMPLEX NETWORKS OF A COMPRESSION ALGORITHM}

We explore complex network representations of the output of a compression algorithm. Our proposed approach begins by digitizing the time series into a set of symbols. Here, to introduce our ideas we only consider binary encoding, i.e., $\{0,1\}$-symbols, where a time series value is converted to 0 if the value is less than the median of the data and is converted to a 1 otherwise. We then compress the symbolic time series using a Lempel-Ziv-Welch-like compression algorithm ${ }^{17}$. This process generates a dictionary of codewords consisting of novel sequences within the symbol sequence and an emitted time series whose values play the role as pointers to the codewords in the dictionary. We treat each codeword in the dictionary as a network node and link two nodes if their pointers in the emitted time series follow each other. We refer to the resulting network as a compression network. The compression network summarizes the temporal dynamical relationship between the compression codewords, thus it will necessarily be of a smaller scale than the original time series. Furthermore, for a binary symbolization, it can be analytically proved that the compression achieved is related to the entropy of the underlying source ${ }^{23}$. We also note that the above wiring procedure is similar to the construction of transition networks. In this case the states (nodes) of the transition networks are the codewords and the links are defined by the emitted time series.

The specific compression algorithm we use to construct the dictionary of codewords, emitted codeword (pointer) sequence and corresponding compression network is as follows. We digitize the time series, say above/below median for an alphabet $A=\{0,1\}$ and initialize the dictionary of codewords with the alphabet symbols. Now, let $p$ be the first symbol of the digitized time series and $q$ the next symbol. We repeat the following steps until we have seen all of the symbolic time series: If $p q$ is not a codeword, i.e., $p q$ is not in the dictionary of codewords, then add $p q$ to the codeword dictionary and emit the codeword pointer corresponding to $p$. Next, set $p=q$, and replace $q$ by the next symbol in the time series. If $p q$ is already a codeword in the dictionary, set $p=p q$ and get $q$ the next symbol in the time series. We continue parsing the symbolic time series in this way, adding new codewords and emitting pointers to codewords until we have seen the entire time series. An explicit example demonstrating the technique on a short sequence is provided in Appendix A. From this, the sufficiently interested reader may confirm the procedure describe here. The result of this process is a dictionary of codewords, indexed by their pointer and an emitted time series of codeword pointers. The dictionary of codewords is built up by adding novel sequences of 0's and 1's as they appear in the (digitized) time series. We observe that not all dictionary codewords will be emitted in the time series of pointers and will demonstrate later that the number of these unused codewords is useful in surrogate data hypothesis tests.

If we regard each codeword in the dictionary as a network node and connect two nodes by a link if their pointers in the emitted time series occur in succession we then obtain a compression network. We now ask what does this network representation of a compression algorithm look like? We show that time series from different underlying processes give rise to different classes of compression networks. That is, structures within the resulting compression networks have different characteristics depending on the underlying process generating the time series. Thus, the structure of compression networks can be used to distinguish different dynamical behaviours.

\section{EXAMPLES}

\section{A. Nonlinear discriminating statistic}

A result of an operational compression algorithm as described above is an emitted sequence of pointers or labels corresponding to entries in a dictionary of codewords. The emitted sequence is a time series which we use to construct a compression network. Not all codewords in the dictionary correspond to a value in the emitted sequence. These are codewords which are seen by the compression algorithm (and hence creating a dictionary entry), but are not seen again in the digitized time series. The number of these unused codewords provides a zeroth level characterization of the time series. We expect uncorrelated random time series to produce a high proportion of unused codewords compared to time series from deterministic systems. A complexity measure based on the number of codewords extracted from a time series has been suggested as a useful quantity with which to characterize the underlying system ${ }^{20}$. Here, we suggest rather, that the ratio, or percentage, of unused codewords in the dictionary is a useful quantity to help to characterize a time series. Although, neither complexity nor our suggestion of proportion of unused codewords are dynamical invariants - they depend on the specific time series ${ }^{24}$ we demonstrate that the ratio of unused codewords can be used as a discriminating statistic in surrogate data hypothesis testing. We study five test systems:

1. uncorrelated Gaussian noise where $x_{t} \sim N(0,1)$.

2. an $\operatorname{AR}(2)$ process given by

$$
x_{t}=\gamma_{1} x_{t-1}+\gamma_{2} x_{t-2}+\varepsilon_{t}
$$

where $\left(\gamma_{1}, \gamma_{2}\right)=(0.1,0.8)$ and $\varepsilon_{t} \sim N(0,1)$.

3. a second $\operatorname{AR}(2)$ process with $\left(\gamma_{1}, \gamma_{2}\right)=(0.1,-0.8)$.

4. a scalar observation from the chaotic Ikeda map, i.e., $x_{t}$ in

$$
\begin{aligned}
& x_{t+1}=a+b\left(x_{t} \cos \theta_{t}+y_{t} \sin \theta_{t}\right) \\
& y_{t+1}=b\left(x_{t} \sin \theta_{t}+y_{t} \cos \theta_{t}\right)
\end{aligned}
$$


where $\theta_{t}=k-\eta /\left(1+x_{t}^{2}+y_{t}^{2}\right)$ and $(a, b, k, \eta)=$ $(1.0,0.9,0.4,6.0)$.

5. scalar observations from a chaotic Mackey-Glass delay differential equation, i.e.,

$$
\frac{d x(t)}{d t}=-\gamma x(t)+\beta \frac{x(t-\Delta)}{1+x(t-\Delta)^{n}}
$$

where $n=10, \beta=0.25, \gamma=0.1$ and $\Delta=17$. $(x(t)=0.5$ as the initial condition for $t<0$.)

For each of the five time series of length 2000 data points we generate surrogates consistent with the null hypotheses: Algorithm 0 (uncorrelated noise), Algorithm 1 (linear filtered noise) and Algorithm 2 (monotonic static nonlinear transformation of linearly filtered noise). We use standard algorithms for generating each Monte Carlo surrogate ${ }^{20}$. As a discriminating statistic we use the ratio of unused codewords obtained by applying the compression algorithm to each time series after digitization to $\{0,1\}$-symbols according to the data values being belowabove the median. We use $B=39$ surrogate data sets for each two-sided test corresponding to a significance level of $\alpha=0.05$ and demonstrate that the ratio of unused codewords rejects or fails to reject the null hypothesis as expected (see, Figure 1 where the data value lies outside of the boxplots ${ }^{25}$ representing the full distribution of surrogate values). We have sufficient evidence to reject the null hypothesis if the value of the discriminating statistic calculated for the data is a maximum or a minimum when compared to the values of the discriminating statistic calculated for the surrogate data - that is, if the filled diamond for the data is outside of the box and whiskers.

To demonstrate the robustness of the proposed statistic with respect to noise in the signal we present in Figure 2 an outcome of an experiment with corrupted nonlinear data. We use the noise-free Ikeda signal from Figure 1 and create eight new time series with additive Gaussian noise corruption to produce signals with $100 \mathrm{~dB}, 80 \mathrm{~dB}$, $60 \mathrm{~dB}, 40 \mathrm{~dB}, 20 \mathrm{~dB}, 10 \mathrm{~dB}, 5 \mathrm{~dB}$ and $1 \mathrm{~dB}$ levels of noise. In Figure 2 we show the (graceful) degradation of the unused codewords as a discriminating statistic for Algorithm 2 hypothesis testing. As before each time series is of length 2000 samples and 39 surrogates were realized for each signal for a $\alpha=0.05$ level of significance. We observe that for signals of $10 \mathrm{~dB}$ and lower the unused codeword statistic is unable to reject the Algorithm 2 null hypothesis. For signals with a higher signal to noise ratio the statistic performs admirably.

To demonstrate the robustness of the proposed unused codeword statistic with respect to noise in the signal and symbolization we present in Figure 3 an outcome of an experiment with corrupted nonlinear data. We once more use noise corrupted Ikeda data and consider 1000 realizations at $10 \mathrm{~dB}$ and $5 \mathrm{~dB}$ respectively. We test the sensitivity of the unused codeword statistic when applied to Algorithm 2 surrogate tests. Since we are using Ikeda
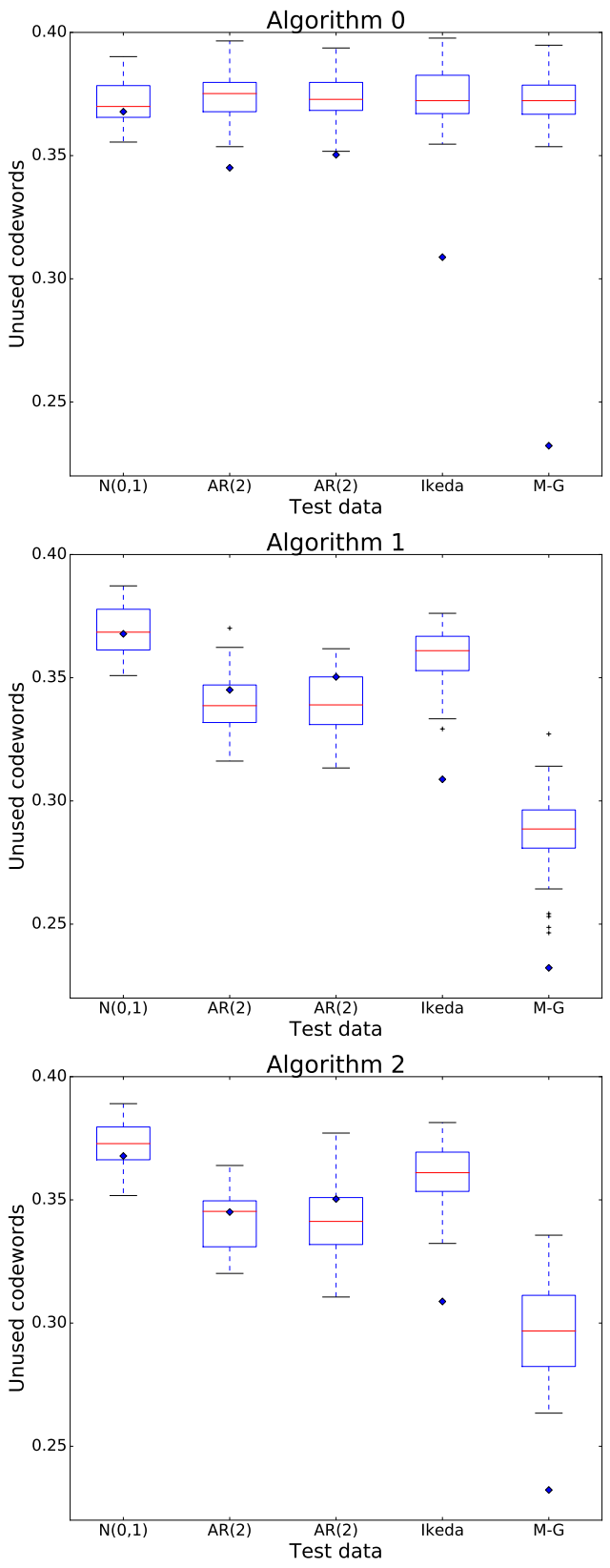

FIG. 1. Unused codewords are a useful discriminating statistic for surrogate tests. Filled diamonds are the data value and the boxplot indicates the distribution of the statistics for $B=39$ surrogate data sets. We see that, as we should, the respective null hypotheses can be correctly rejected or not at $\alpha=0.05$ level of significance.

data, the sensitivity estimates the probability of failing to reject the null hypothesis when we should reject it, i.e., a measure of type II errors. To compare different symbolizations we consider time series of variable length such that the ratio of the length of the time series to the number of symbols is a constant. We digitize the time series using percentiles and consider 2, 4, 6, 8 and 10 symbols. We select the aforementioned ratio to be equal 


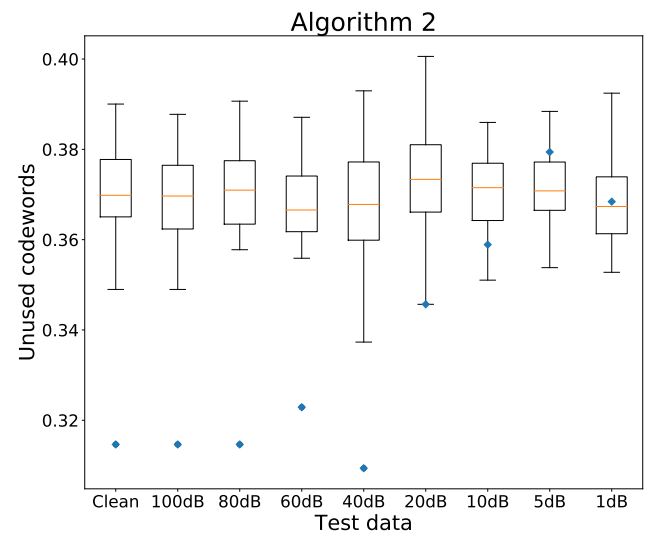

FIG. 2. Robustness of unused codewords with respect to noise. Filled diamonds are the data value and the boxplot indicates the distribution of the statistics for $B=39$ surrogate data for each Ikeda data set. We see that the null hypotheses can be correctly rejected at $\alpha=0.05$ level of significance for signals with a signal to noise ratio of above $10 \mathrm{~dB}$.

to 1000 , so that the length of the time series for binary symbolization is $N=2000$ as before, for four symbols $N=4000$ etc. We note that for clean Ikeda data the ability of the unused codewords to reject the null hypothesis is unaffected by the length of symbolization, i.e., the sensitivity is zero for all symbolizations tested. In Figure 3 we show the results of increasing the number of symbols for noise corrupted Ikeda data. We see that in the $10 \mathrm{~dB}$ case increasing the number of symbols beyond two mitigates the effects of the noise and the sensitivity drops to zero. For a higher noise level $-5 \mathrm{~dB}$ - we see that increasing the number of symbols also mitigates the effects of the noise, but performance drops again when using 10 symbols. It is unclear what is responsible for this effect but it highlights that increasing the number of symbols is not a panacea for noisy data. As such in the following we continue to consider binary symbolization for expediency, but recognize that for a particular application increasing the number of symbols is worth examining further.

\section{B. EEG data}

We have shown that the number of unused codewords, or isolated nodes in a compression network, can be a useful discriminating statistic for surrogate data tests. To highlight the usefulness of this statistic beyond mathematical curiosities we consider an EEG signal from the CHB-MIT dataset available at Physionet ${ }^{26}$. The selected signal has a $256 \mathrm{~Hz}$ sample rate with 16 -bit resolution and was chosen because it has a definitive and easily identifiable behavioural transition. In particular, annotations of the beginning and the ending of seizures are available for signals with one or more seizure occurrences. Our goal is to show that surrogate tests with our proposed statistic

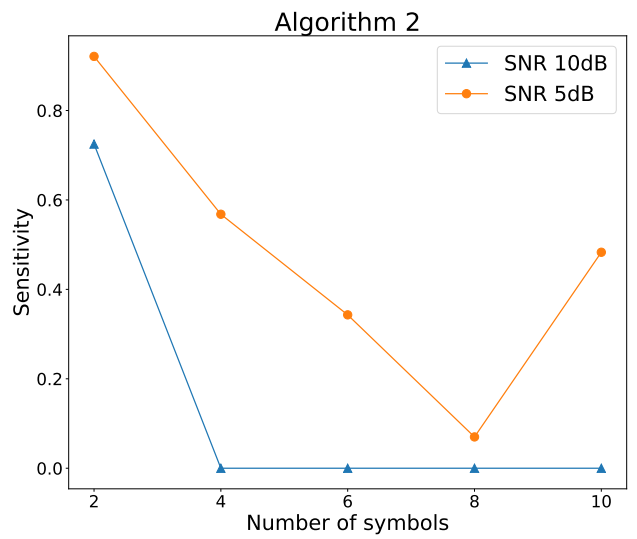

FIG. 3. Sensitivity of unused codewords in Algorithm 2 tests with respect to symbolization of noisy Ikeda data. The ratio [length of time series]:[number of symbols] is held constant, equal to 1000 .

on non-overlapping segments of the data can successfully identify the behavioural transitions present in the data.

Many studies have indicated that a typical EEG channel will present a gradual transition between brain states, for instance, preictal state is usually referred to as the period from the interictal (periods of seizure free intervals other than the preictal) to ictal (during) seizure states ${ }^{27}$. Algorithmic seizure prediction models have thus considered the seizure prediction as an early detection of the preictal stage, and it has been shown that this stage indeed presents distinct characteristics from the interic$\mathrm{tal}^{28,29}$. Moreover, the potential detection of the preictal stage is important as the notion of intervention time (defined as in between the end of the preictal stage and the seizure onset) can be applied.

From a nonlinear dynamics perspective, studies have indicated changes in the underlying dynamics in the minutes before the epileptic seizure, for example, Iasemidis and colleagues pointed out the preictal stage presents a decrease in chaoticity ${ }^{30}$. Others indicated that such changes can also be observed in terms of a decrease in the amplitude distribution of the permutation entropy ${ }^{31}$, or a decrease in the spatiotemporal complexity ${ }^{32}$.

The result of our method applied to this EEG signal with a clear behavioural change is shown in Figure 4. The analysis was conducted in a moving time-window of 60 seconds with no overlap. In each 60 s segment thirty nine constrained Algorithm 2 surrogate data sets were realized. While other window lengths were verified, our method highlighted specific changes in the present patterns over long-term segments of 60 seconds.

The lower panel of Figure 4 shows that our unused codeword statistic was also useful to detect two behavioral regime changes. The first prominent behavioral change can be observed at around sample 500,000 and time window 30 . This change corresponds to approximately 20 minutes before the seizure onset according to the annotation available for the data. Studies have 
adopted preictal times from $2 \mathrm{~min}$ to $90 \mathrm{~min}^{27}$ in the literature. While there is not a preictal annotation for this signal, our statistic may be indicating a change that is related to the predisposition of a seizure.

For the signal depicted in Figure 4, a seizure was annotated as occurring between samples 766,976 and 777,216. Our statistic clearly indicates a second prominent change in the signal, as one can observe the rejection of the null hypothesis at window 52. Thus, despite the number of unused codewords being non-pivotal (as a surrogate test statistic) it appears to possess a precise enough resolution to distinguish behavioural change in EEG signals with respect to standard surrogate generation methods.
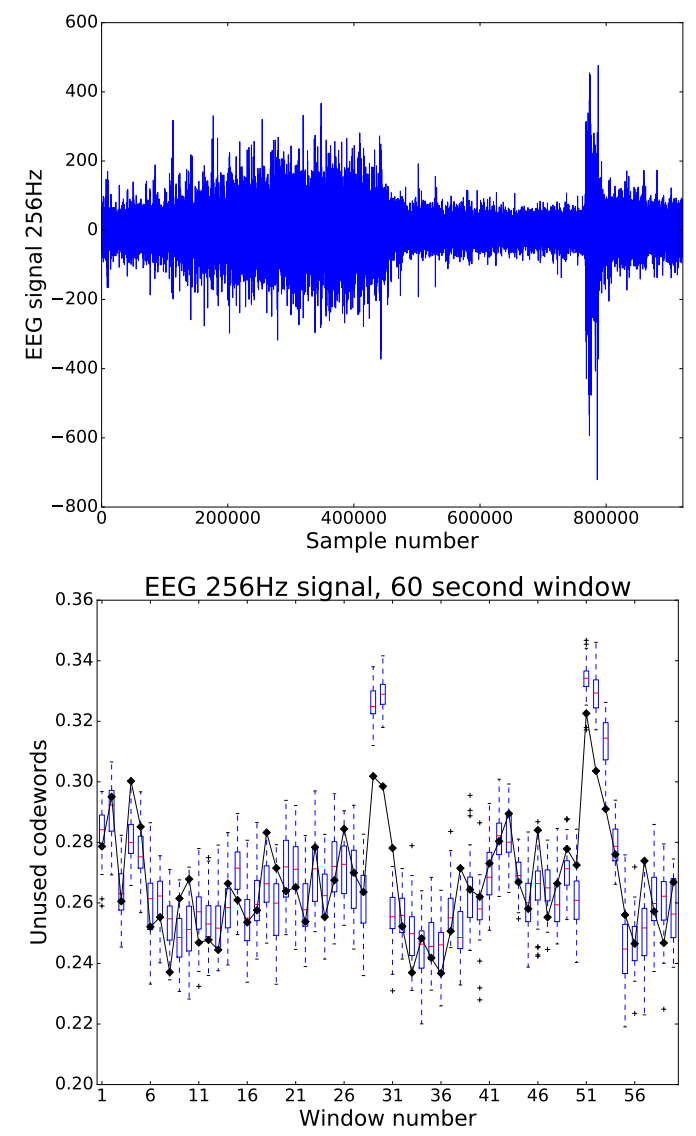

FIG. 4. (upper panel) The EEG time series at $256 \mathrm{~Hz}$ sampling demonstrably exhibits two behavioural regime changes. (lower panel) Unused codewords detect onset of change in EEG scans. Connected filled diamonds are the data value and the boxplot indicates the distribution of the statistics for $B=39$ algorithm 2 surrogate data sets. A non-overlapping sliding window of 60 seconds constituted the data time series. A binary encoding based on the median of the data was the basis for symbolization. We see that, although within EEG behavioural regime there is a failure to reject the null hypothesis at significance level $\alpha=0.05$, the onset of behavioural change was detected by the unused codeword statistic and furthermore corresponded to rejection of the null hypothesis.

\section{Chaotic systems}

The percentage of unused codewords can be regarded as a zeroth level characterization of a time series. The emitted sequence of codewords and the compression network it induces can be studied to further characterize the system. This provides a novel perspective to analyze properties of the compression network with respect to time series. We now turn attention to the topological properties of the largest connected component of a binary reduction of the compression network, i.e., the properties of the network constructed with the used codewords as network nodes and network links defined by the sequential transitions of the emitted sequence.

As previously mentioned the compression achieved by the compression algorithm using a binary symbolization is asymptotic to the entropy of the underlying source. We show how this result manifests itself in the size of the compression network's largest connected component in terms of number of nodes. Consider the logistic map $x_{n+1}=\lambda x_{n}\left(1-x_{n}\right)$ for $x_{0} \in(0,1)$ and the parameter $\lambda \in[3.4,4.0]$. For a given value of $\lambda$ we iterate the logistic map from a random initial condition and retain an orbit of length 2000 points after discarding an initial transient. The familiar bifurcation diagram is shown in the upper panel of Figure 5. The central panel shows the sample entropy $^{33}$ and a numerical evaluation of the Lyapunov exponent along the orbit using the analytical expression for the derivative. The network size in the lower panel is represented by the number of nodes in the largest connected component normalized by the length of the time series. The ratio of unused codewords is the statistic proposed earlier and is also shown in the lower panel. Qualitative similarities between the sample entropy, the network size and the ratio of unused codewords is apparent in the chaotic regimes.

We further describe the capacity of the compression networks to capture changing system behaviour for short time series. Consider, the Rössler system of ordinary differential equations ${ }^{34}$ whose behaviour changes with respect to a bifurcation parameter $c$ to produce the bifurcation diagram shown in the upper panel of Figure 6. We observe windows of periodic behaviour of different periods and more complicated behaviour including chaos. The bifurcation diagram was generated for each value of $c$ by integrating the differential equations with a time step of 0.15 , observing the $x$-coordinate of the system and retaining 4000 data points after discarding an initial transient. In the chaotic regime, extracting the peaks of this output we obtain a time series on an approximate Poincaré section of length 100. These time series can be transformed to a compression network using our proposed protocol, i.e., digitize to $\{0,1\}$-symbols, apply the compression algorithm and construct the compression network from the emitted sequence of codewords. Despite the short length of these maxima time series, properties of the resulting compression networks reflect the changing dynamical behaviour as the bifurcation pa- 


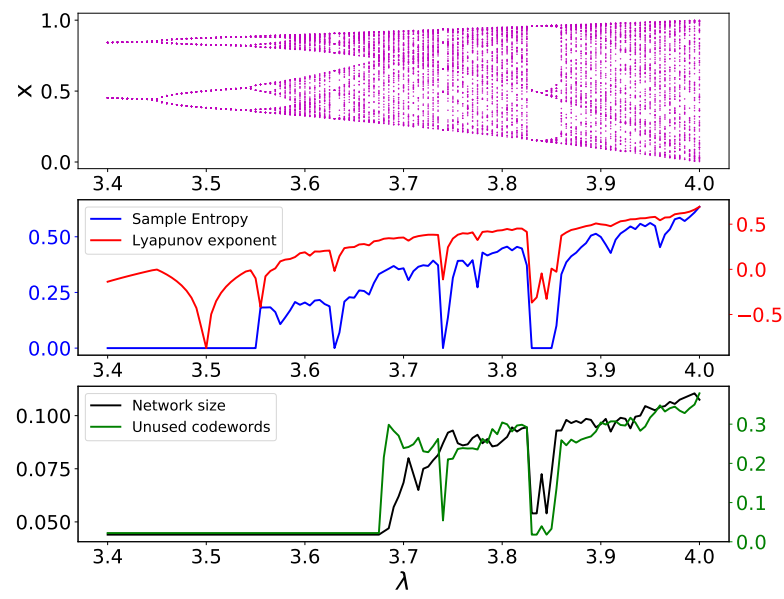

FIG. 5. (upper panel) Bifurcation diagram of the logistic map with respect to the parameter $\lambda$. (central panel) Sample entropy calculated using the python nolds module and the Lyapunov exponent calculated along the trajectory using the analytical expression for the derivative. (lower panel) Number of nodes in the largest connected component of the network normalized by the length of the time series and the ratio of unused codewords. The size of the network and the ratio of unused codewords show qualitatively similar features to dynamical invariants of the signal.

rameter $c$ is changed (lower panel of Figure 6.) In this case we have selected a count of the number of cycles, including self-loops, in a binary reduction of the networks. That is, we have converted the weighted, directed compression network to an unweighted, undirected network. Recall, a network cycle is a closed walk consisting of a sequence of nodes starting and ending at the same node, with two consecutive nodes in the sequence adjacent to each other in the network.

We see that the compression networks corresponding to periodic behaviour typically have a simple structure in terms of network cycles (e.g., the compression network corresponding to the period two orbit at $c=6$ has zero cycles and so is acyclic). We contrast such simple structural connectivity to the more intricate connections - higher number of cycles - for more complicated behaviour. For example, in Figure 7 the period four orbit at $c=8.2$ consists of a simple line structure and two isolated nodes corresponding to the unused codewords. Chaotic behaviour observed at $c=17$ results in a compression network with more unused codewords (i.e. isolated nodes) and some cyclic connectivity.

The attentive reader will note that for some periodic windows the structure of the binary reduced network in terms of network cycles is more complicated than anticipated from the preceding discussion. For a periodic signal our tests suggest the resulting compression network should be acyclic. The discrepancy is easily explained by our naive imposition of a binary symbol encoding. For period- $n$ data, an $n$-ary encoding is clearly optimal. This would lead to the expected acyclic network. Here, we have intentionally applied a binary symbolic encoding for all behaviours (in the bifurcation diagram) in order to warn of the dangers of over-interpreting the results of one encoding and one summary network property. In short, the compression networks which we construct are a characterization of the symbolic encoding of the original time series. Different encodings will naturally yield different information.
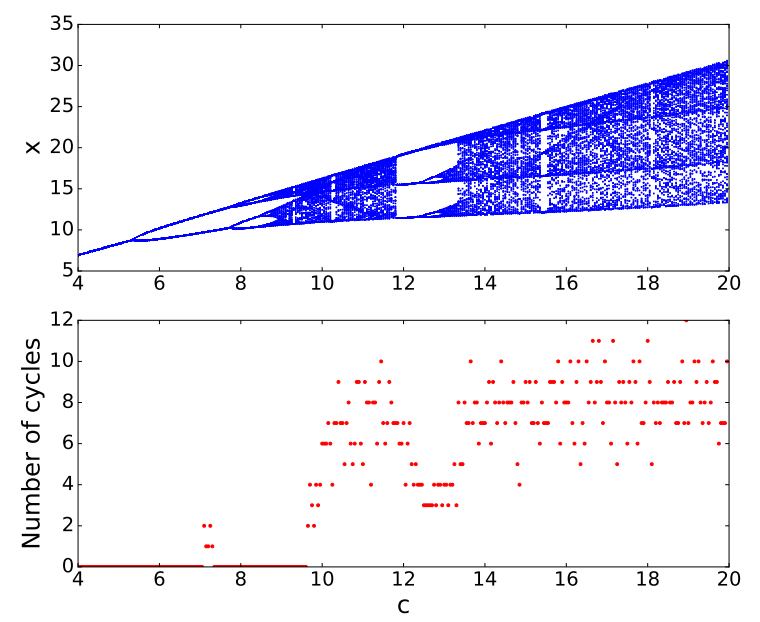

FIG. 6. Compression network properties capture system change. The upper panel depicts the bifurcation of the Rössler system with respect to the parameter $c$. The lower panel shows the number of cycles in the corresponding compression network representation with respect to a binary encoding (self-loops within the compression networks are counted as cycles.) In general, the chaotic windows correspond to more complex compression network representations, i.e., more cycles, in contrast to the simpler structures within periodic windows. However, the regions of period-3 dynamics appear somewhat overly complex: as described in the text.

As demonstrated above the cycles in the largest connected component of the compression network are a rich summary of the topological properties of the network. A cycle of length $k$ can be considered as the number of new codewords seen before an older codeword recurs. Thus the $k$-cycle distribution provides detailed information about the compressibility of the time series. Furthermore the $k$-cycle distribution contains information equivalent to other network measures, for example, clustering coefficient is related to the number of 3-cycles, square clustering to the number of 4-cycles. We can calculate the distribution of $k$-cycles in a network by calculating a minimal cycle basis of the network $\mathrm{k}^{35}$ and use this distribution to help distinguish between different types of dynamics. To illustrate, we consider 1000 realizations of i.i.d. $N(0,1)$ noise each of length 2000 and 1000 orbits of length 2000 of the Ikeda map distinguished by a random initial condition. For each realization and orbit we perform a binary symbolization of the time series and apply the compression algorithm. We extract the largest connected component of the resulting compression network and calculate a minimal cycle basis for it. In Figure 8 we 


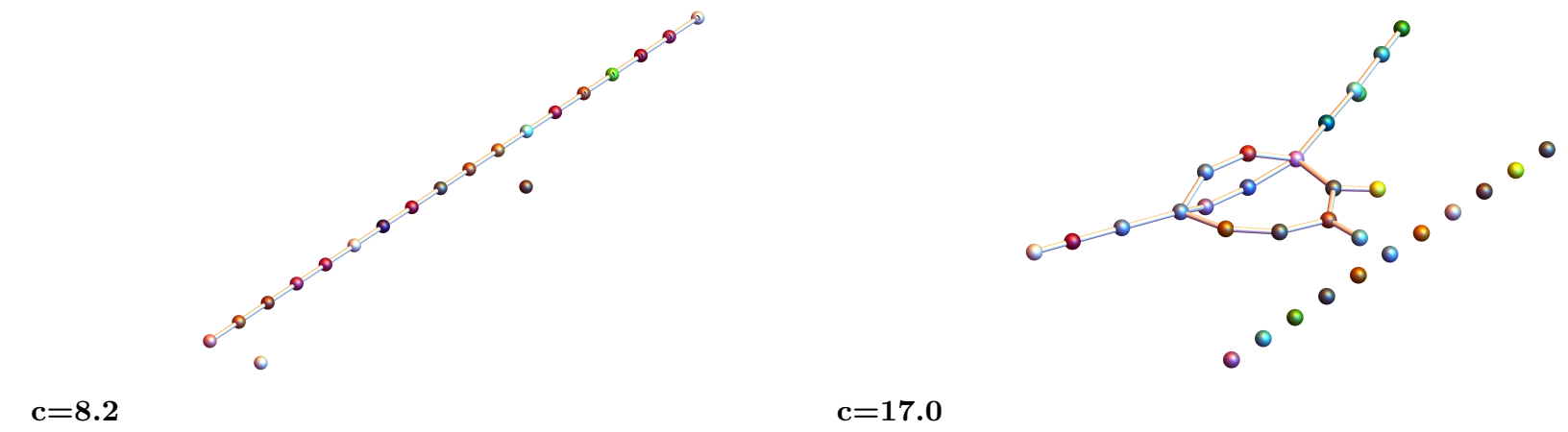

FIG. 7. Compression network structure for (left panel) periodic data at $c=8.2$ and (right panel) chaotic data at $c=17.0$. Self-loops are not drawn but are counted in Figure 6. Unused codewords are represented by isolated (degree zero) nodes. Note that in both cases the network structure represents a substantial simplification of the underlying dynamical behaviour. Of course, the network is only built from the binary quantization of the original continuous valued signal.

show the $k$-cycle distributions for i.i.d. noise and Ikeda map data. The symbols show the mean count of cycles of length $k$ over the 1000 time series and the bars extend to one standard deviation. A visual inspection suggests differences between the topological properties of i.i.d. noise and chaotic data. We can quantify this difference and gauge if the visual differences are significant. Examining each $k$-cycle distribution separately, i.e., comparing the distribution of the number of 3-cycles obtained for i.i.d. noise and Ikeda data using a Kolmogorov-Smirnov test, we find that the p-value of this test is much lower than 0.05 , indicating the two distributions are different. We find similar results for all $k$-cycles, $3 \leq k \leq 18$ except for $k=9$. Thus we have evidence that the topological properties of the largest connected components of the compression networks can help distinguish between random and chaotic dynamics.

\section{SUMMARY}

In this paper we have proposed a method of representing the results of a sequential compression algorithm as a complex network. The structure of (a binary reduction of) the resulting network appears capable of distinguishing different dynamical behaviour of the underlying system can also distinguish between random verses nonlinear dynamics using chaotic systems. Furthermore, as an unexpected byproduct, a quantity based on the number of unused codewords, or isolated (degree zero) nodes in the network can be usefully applied as a discriminating statistic in surrogate data sets. We demonstrated such for different underlying systems, showing the statistic can reject, or fail to reject, null hypotheses as appropriate and is robust to high levels of noise corruption. Moreover, the statistic proved useful in identifying behavioural change within an experimental EEG signal. We suggest that considering the structure of complex network representations of compression algorithms is a promising framework

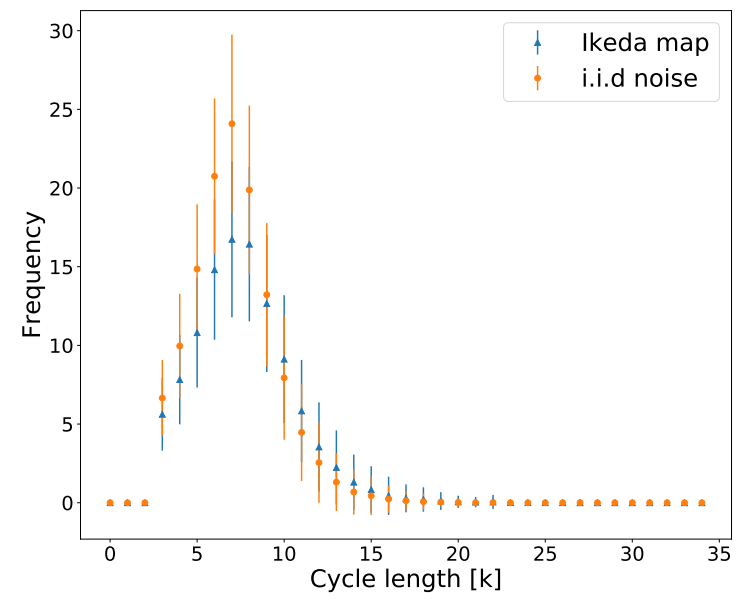

FIG. 8. Length distributions of minimal cycle bases of the largest connected component of compression networks for i.i.d. noise and chaotic Ikeda data. There is evidence via a Kolmogorov-Smirnov test that the distributions of $k$-cycles are significantly different at 0.05 level indicating that the topological structure of compression networks can distinguish between different types of dynamics.

for further nonlinear time series analysis.

\section{ACKNOWLEDGMENTS}

MS is supported by the Australian Research Council through Discovery Project DP140100203.

${ }^{1}$ J. Donges, Y. Zou, N. Marwan, and J. Kurths, Europhys. Lett. 87, 48007 (2009).

${ }^{2}$ M. Galbiati, D. Delpini, and S. Battiston, Nat. Phys. 9, 126 (2013).

${ }^{3}$ M. S. Hossain and A. Fourie, Géotechnique 63, 641 (2012).

${ }^{4}$ J. Wang, K. Zhang, L. Xu, and E. K. Wang, Proc. Natl. Acad. Sci. USA 108, 8257 (2011).

${ }^{5}$ R. M. May, Nature 269, 471 (1977).

${ }^{6}$ J. Zhang and M. Small, Phys. Rev. Lett. 96, 238701 (2006). 
${ }^{7}$ L. Lacasa, B. Luque, F. Ballesteros, J. L. J, and J. N. no, Proc. Natl. Acad. Sci. USA 105, 4972 (2008).

${ }^{8}$ B. Luque, L. Lacasa, F. Ballesteros, and J. Luque, Phys. Rev. E 80, 046103 (2009).

${ }^{9}$ X.-K. Xu, J. Zhang, and M. Small, Proc. Natl. Acad. Sci. USA 105, 19601 (2008).

${ }^{10}$ N. Marwan, J. F. Donges, Y. Zou, R. V. Donner, and J. Kurths, Phys. Lett. A 373, 4246 (2009).

${ }^{11}$ R. V. Donner, M. Small, J. F. Donges, N. Marwan, Y. Zou, R. Xiang, and J. Kurths, Inter. J. of Bifur. and Chaos 21, 1019 (2011)

${ }^{12}$ M. McCullough, M. Small, T. Stemler, and H.-C. Iu, Chaos 25, 053101 (2015).

${ }^{13}$ A. Tordesillas, D. M. Walker, E. Andò, and G. Viggiani, Proc. Roy. Soc. Lond. Ser. A 469, 20120606 (2013).

${ }^{14}$ J. Rissanen, Stochastic Complexity in Statistical Inquiry, Series in Computer Science, Vol. 15 (World Scientific, Singapore, 1989).

${ }^{15}$ K. Judd and A. Mees, Physica D: Nonlinear Phenomena 82, 426 (1995).

${ }^{16}$ K. Judd and A. Mees, Physica D: Nonlinear Phenomena 120, 273 (1998).

${ }^{17}$ T. Welch, Computer 17, 8 (1984).

${ }^{18}$ D. J. C. MacKay, Information Theory, Inference, and Learning Algorithms (Cambridge University Press, 2003).

${ }^{19}$ J. Theiler and D. Prichard, Physica D: Nonlinear Phenomena 94, 221 (1996).

${ }^{20}$ M. Small, Applied Nonlinear Time Series Analysis: Applications in Physics, Physiology and Finance, Nonlinear Science Series A, vol. 52 (World Scientific, Singapore, 2005).

${ }^{21}$ J. Theiler, S. Eubank, A. Longtin, B. Galdrikian, and J. D. Farmer, Physica D: Nonlinear Phenomena 58, 77 (1992).

${ }^{22}$ M. Small and K. Judd, Physica D: Nonlinear Phenomena 120, 386 (1998).

${ }^{23}$ T. M. Cover and J. A. Thomas, Elements of Information Theory (John Wiley \& Sons Inc, New Jersey, 2006).

${ }^{24}$ Or more, precisely, they depend on the observation function and are hence not invariant under smooth rescaling of the data.

${ }^{25}$ Boxplots illustrate the distribution of a statistic by partitioning observations into four equally populated sections. The red central line indicates the median, and the box which surrounds it represents the central $50 \%$ of values. The vertical lines (whiskers) span the values above and below the boxed interval.

${ }^{26}$ https://physionet.org/physiobank/database/chbmit/.

${ }^{27}$ E. B. Assi, D. K. Nguyen, S. Rihana, and M. Sawan, Biomedical Signal Processing and Control 34, 144 (2017).

${ }^{28}$ K. Gadhoumi, J. M. Lina, F. Mormann, and J. Gotman, Journal of neuroscience methods 260, 270 (2016).

${ }^{29} \mathrm{~K}$. Lehnertz, In Engineering in Medicine and Biology Society Proceedings of the IEEE 23rd Annual International Conference 4, 4121 (2001).

${ }^{30}$ L. D. Iasemidis, J. C. Sackellares, H. P. Zaveri, and W. J. Williams, Brain topography 2, 187 (1990).

${ }^{31}$ A. A. Bruzzo, B. Gesierich, M. Santi, C. A. Tassinari, N. Birbaumer, and G. Rubboli, Neurological Sciences 29, 3 (2008).

${ }^{32}$ J. Martinerie, C. Adam, M. L. V. Quyen, M. Baulac, S. Clemenceau, B. Renault, and F. J. Varela, Nature medicine 4, 1173 (1998).

${ }^{33}$ Approximated using the nolds python module.

${ }^{34}$ O. Rossler, Phys. Lett. A 57, 397 (1976).

${ }^{35}$ K. Mehlhorn and D. Michail, J. Exp. Algorithm. 11, 1 (2006).

${ }^{36}$ N. Moghim and D. W. Corne, PLoS One 9, e99334 (2014).

\section{Appendix A: Example of the operation of the compression algorithm}

The compression algorithm used to transform the time series to a complex network is a Lempel-Ziv-Welch-like method. We work with the quantized time series and pro- ceed as indicated in Figure 9. Consider the symbolic time series $s=111011001100011$ - obtained from fourteen iterates of the logistic map with $\lambda=4, x_{0}=0.8173$ and a symbolic encoding where $x \leq \frac{1}{2}$ gives $s=0$ and $s=1$ otherwise. We initially add the symbol alphabet to the codeword dictionary $(C D)$. Thus, $C D=\{0: 0,1: 1\}$, where 0 is the label for symbol 0 and 1 is the label for symbol 1. Next we set the first symbol of the time series as $p$, so that $p=1$ and set as $q$ the next symbol in the time series, i.e., $q=1$. We concatenate $p$ and $q$ to form $p q=11$ and check if $p q$ is already a codeword, i.e., if it is already included in the dictionary.

If $p q$ is not in the dictionary then $p q$ is a novel codeword. We extend the dictionary to include $p q$ and start a new emitted time series (say, $T S$ ) by emitting $p$. Thus, the codeword dictionary becomes $C D=\{0: 0,1: 1,2$ : $11\}$, where we have given the codeword 11 the label 2 . The emitted time series is $T S=\{1\}$ since $p=1$ is a codeword with label 1 . We now step along the symbolic time series by setting $p=q$ and setting $q$ as the next symbol. For this step, $p=1$ is the second symbol in the time series, and $q=1$ the third symbol in the time series.

If $p q$ is not a novel codeword, i.e., it is already in the dictionary, then we proceed differently. This arises at the current situation. We have $p=1$ and $q=1$ so $p q=11$. We have just added codeword 11 to the dictionary and so it is not novel. Therefore, we set $p=p q$ and let $q$ be the next symbol in the time series. That is, $p=11$ and $q=0$ the fourth symbol in the quantized time series. Now, we concatenate $p$ and $q$ and check if $p q=110$ is a novel codeword; it is. So, add 110 to the dictionary and emit the codeword label corresponding to $p=11$. The dictionary has now been updated to $C D=\{0: 0,1: 1,2$ : $11,3: 110\}$ and the emitted time series is $T S=\{1,2\}$. We update $p$ to be $p=q=0$ - the fourth symbol in the time series - and let $q=1$ be the next symbol (the fifth) in the time series.

We continue in the above fashion, scanning through the symbolic time series, updating the dictionary of codewords when we see a novel codeword and emitting the codeword labels to form a new time series. When we reach the end of the time series we emit a final codeword label corresponding to the dictionary codeword that matches our current $p q$ symbol sequence. For the time series in this description the final codeword dictionary and emitted time series are shown in Figure 9.

We observe that some codewords are introduced to the dictionary but do not appear in the emitted time series. These are codewords which are seen once in the symbolic time series but are not seen again and so they are not a prefix code of another novel sequence.

We can also see how this Lempel-Ziv-Welch-like algorithm achieves compression for longer symbolic time series. Although the codewords are of variable length and becoming longer, they are replaced by smaller labels in the emitted time series. The length of the emitted time series is also much shorter than the original time series and, since the dictionary only needs to be communicated 
once, we would expect the code length of [dictionary + emitted time series] to be shorter than the code length of [original symbolic time series].

In the complex network representation, nodes represent the codewords of the emitted time series and two nodes are linked whenever one codeword follows the other in the codeword sequence. For the example used in this description, Figure 9 also presents the resulting compression network. The isolated nodes represent the codewords which were not used, i.e., do not appear in the emitted time series. 


\section{Symbolic time series: $s=111011001100011$}

Start a dictionary of codewords by adding the symbol alphabet. Begin scanning the time series:

Set $p=1$ and $q=1, s=111011001100011$

$\mathrm{pq}=11$ is novel $->$ add $\mathrm{pq}$ as codeword and emit codeword $\mathrm{p}$ label.

Set $p=q=1$ and let $q=1$ be next symbol, i.e.,

$s=111011001100011$

$p q=11$ is not novel $->$ set $p=p q$ and let $q=0$ be next symbol, i.e., $s=111011001100011$

$p q=110$ is novel $->$ add $p q$ as codeword and emit codeword $p$ label.

Set $p=q=0$ and let $q=1$ be next symbol, i.e.,

$s=111011001100011$

$p q=01$ is novel $->$ add pq as codeword and emit codeword $p$ label.

Continuing in this fashion we achieve the codeword dictionary and the emitted time series of codeword labels.

(When we reach the end of the symbolic time series we emit the codeword label corresponding to the final pq.)

\section{Codeword dictionary:}

\begin{tabular}{lll} 
Codeword label & Codeword \\
\hline 0 & 0 \\
1 & 1 \\
2 & 11 \\
3 & 110 \\
4 & 01 \\
5 & 1100 \\
6 & 011 \\
7 & 10 \\
8 & 00 \\
9 & 001 \\
\hline Emitted time series: & & \\
$\{1,2,0,3,4,1,0,8,2\}$ & & \\
& 6 &
\end{tabular}

FIG. 9. The initial steps of the compression algorithm together with the final dictionary of codewords, the emitted time series of codeword labels and the resulted compression network. (Colours indicate the progression through the time series.) 